\title{
Increased expression of the immune modulatory molecule PD- L1 (CD274) in anaplastic meningioma
}

\author{
Ziming Du ${ }^{1}$, Malak Abedalthagafi ${ }^{1}$, Ayal A. Aizer ${ }^{2}$, Allison R. McHenry ${ }^{3}$, Heather \\ H. Sun ${ }^{1}$, Mark-Anthony Bray ${ }^{4}$ Omar Viramontes ${ }^{5}$, Revaz Machaidze ${ }^{1,5}$, Priscilla \\ K. Brastianos ${ }^{6,7}$, David A. Reardon ${ }^{6}$, Ian F. Dunn ${ }^{5}$, Gordon J. Freeman ${ }^{6}$, Keith \\ L. Ligon ${ }^{1,6}$, Anne E. Carpenter ${ }^{4}$, Brian M. Alexander ${ }^{2}$, Nathalie Y. Agar ${ }^{5}$, Scott $\mathrm{J}$. \\ Rodig $^{1}$, Elizabeth M. Bradshaw ${ }^{3}$ and Sandro Santagata ${ }^{1,8}$ \\ ${ }^{1}$ Department of Pathology, Brigham and Women's Hospital and Harvard Medical School, Boston, Massachusetts, USA \\ 2 Department of Radiation Oncology, Brigham and Women's Hospital and Harvard Medical School, Boston, Massachusetts, \\ USA \\ ${ }^{3}$ Department of Neurology, Brigham and Women's Hospital and Harvard Medical School, Boston, Massachusetts, USA \\ ${ }^{4}$ Imaging Platform, The Broad Institute of MIT and Harvard, Cambridge, Massachusetts, USA \\ ${ }^{5}$ Department of Neurosurgery, Brigham and Women's Hospital and Harvard Medical School, Boston, Massachusetts, USA \\ ${ }^{6}$ Department of Medical Oncology, Dana-Farber Cancer Institute and Harvard Medical School, Boston, Massachusetts, USA \\ 7 Department of Neuro-Oncology, Massachusetts General Hospital and Harvard Medical School, Boston, Massachusetts, USA \\ ${ }^{8}$ Department of Cancer Biology, Dana-Farber Cancer Institute and Harvard Medical School, Boston, Massachusetts, USA \\ Correspondence to: Sandro Santagata, email: ssantagata@partners.org
}

Keywords: meningioma, PD-L1, RNAscope, immunotherapy

Received: December 15,2014 Accepted: December 28, $2014 \quad$ Published: December 31, 2014

This is an open-access article distributed under the terms of the Creative Commons Attribution License, which permits unrestricted use, distribution, and reproduction in any medium, provided the original author and source are credited.

\section{ABSTRACT}

There are no effective medical treatments for WHO grade III (anaplastic) meningioma. Patients with this high-grade malignancy have a median survival of less than two years. Therapeutics that modulate the mechanisms that inhibit local immune responses in the tumor microenvironment are showing significant and durable clinical responses in patients with treatment refractory high-grade tumors. We examined the immune infiltrate of $\mathbf{2 9 1}$ meningiomas including WHO grade I-III meningiomas using immunohistochemistry and we examined the expression of PD-L1 mRNA by RNAscope in situ hybridization and PD-L1 protein by immunohistochemistry. In meningioma, the tumor infiltrating lymphocytes are predominantly $\mathrm{T}$ cells. In anaplastic meningioma, there is a sharp decrease in the number of T cells, including the numbers of CD4+ and CD8 + T cells and cells expressing PD-1 and there is also an increase in the number of FOXP3 expressing immunoregulatory (Treg) cells. PD-L1 expression is increased in anaplastic meningioma - both mRNA and protein. Using patient derived meningioma cell, we confirm that PD-L1 is expressed in meningioma cells themselves, and not solely in infiltrating immune cells. This work indicates that high-grade meningioma harbor an immunosuppressive tumor microenviroment and that increased Treg cells and elevated PD-L1 may contribute to the aggressive phenotype of these tumors.

\section{INTRODUCTION}

Meningiomas are the most common primary intracranial tumor accounting for over one third of all brain tumors [1]. In the United States, the incidence of pathologically-confirmed meningioma is approximately
7.4 per 100,000 individuals with a prevalence of 97.5 per 100,000 individuals - there are approximately 18,000 new cases diagnosed annually and 170,000 people living with a diagnosis of meningioma $[1,2]$. Most meningioma are WHO grade I tumors and can be treated effectively with surgery, however, a subset have more aggressive features. 
Over $20 \%$ of meningioma are WHO grade II (atypical) tumors [3] and approximately 3\% are WHO grade III (anaplastic) meningioma [4]. Patients with WHO grade II or III meningiomas are significantly more likely to have a local recurrence after their initial treatment and moreover have a shorter overall survival compared to patients with WHO grade I meningioma [5]. Reported recurrence rates vary widely across published reports but there is a strong association of recurrence with WHO grade: 7-20\% for WHO grade I meningioma, 30-40\% for atypical meningioma and 50-94\% for anaplastic meningioma [3, 6-9]. Notably, the prognosis for anaplastic meningioma is much worse than for atypical meningioma - in one large study atypical meningioma had a 5-year mortality rate of $21 \%$ while anaplastic meningioma had a 5-year mortality rate of $68 \%$ with a median survival of only 1.5 years [6].

Options for blocking meningioma growth with targeted therapeutics appear possible for only a small subset of patients - for example, selective inhibitors of SMO and AKT may prove to be useful for patients with tumors harboring driver mutations in those oncogenes. Such meningiomas appear to be most frequently WHO grade I tumors that arise in the skull base $[10,11]$. Meningiomas also frequently harbor mutations in NF2 [12-16] as well as in TRAF7 and in KLF4 [10] [17]. A subset of meningioma (the angiomatous subtype) harbor chromosomal polysomies [18] and the clear cell subtype can have mutations in SMARCE1 [19]. High-grade meningioma characteristically bear recurrent whole arm chromosomal losses [20-24] and TERT promoter mutations associated with histological progression [25]. Because none of these additional genetic aberrations can currently be targeted with selective therapeutics, strategies using broadly active agents may be needed for effective disease-management in most patients with high-grade meningioma.

Insights into the molecular mechanisms underlying how the immune system responds to tumors has uncovered the important role of immune checkpoint pathways that regulate the function of tumor infiltrating lymphocytes [26-30]. Recent clinical trials of agents targeting PD-1 or PD-L1 have demonstrated durable tumor regression and prolonged stabilization of disease in patients with advanced non-small-cell lung carcinoma, melanoma, renal-cell carcinoma and Hodgkin's lymphoma [31-35].

In light of these findings, and with an interest in finding potential strategies for treating patients with highgrade meningioma, we have characterized the lymphocytic infiltrate of meningioma and demonstrate that there is an immunosuppressive microenvironment in anaplastic meningioma. Because the level of PD-L1 is the most important factor for predicting response to anti-PD-1 blockade [36] we have characterized PD-L1 expression using orthogonal techniques - immunohistochemistry and in situ hybridization with RNAscope [37]. Scoring of 291 cases separated into two cohorts using visual and several digital analysis platforms show that anaplastic meningioma have elevated PD-L1 protein and mRNA levels. This observation raises the possibility of testing immune checkpoint blockade for the immunotherapy of anaplastic meningioma.

\section{RESULTS}

\section{Characterization of the immune infiltrate of meningioma in tissue specimens}

We characterized the lymphocytic infiltrate of meningioma in tissue resection specimens using three tissue microarrays containing samples from a total of 291 cases - 195 WHO grade I, 73 WHO grade II and 23 WHO grade III (see Table S1 for subtype breakdown). We had constructed two of the TMAs (TMA283 \& 285) previously for our study characterizing the mutation profile of meningioma [11]. We produced another TMA (TMA310) consisting of the 118 cases, most $(n=99)$ of which were diagnosed consecutively between 2012 and 2013 by the neuropathology service at Brigham and Women's Hospital. In addition to the standard meningioma subtypes (meningothelial, fibroblastic and transitional), these TMAs also contained cases of several less frequent subtypes (psammomatous, angiomatous, secretory and chordoid meningioma for instance). We stained each of the three TMAs with antibodies that recognize panlymphocyte marker (CD45/LCA), T-lymphocyte markers (CD3, CD4, CD8), B-cell marker (CD20), Treg marker (FOXP3) and immune regulatory marker PD-1 and quantified the data using both visual scoring by light microscopy review (Table S2) and Aperio Imagescope software (Fig. 1, Table 1 and Table S3). Notably, we found very similar results with both independent scoring methods (Table S2 and Table 1).

We found that the lymphocyte infiltrate in the meningioma tissue was comprised predominantly of $\mathrm{T}$ cells $($ median $=14.3 \mathrm{CD} 3+$ cells per core across all grades, Table S4) with infrequent B cells (median $=0.3$ CD20+ cells per core across all grades, Table S4) $(\mathrm{p}<$ $0.0001)$ (Fig. 1A). We noted a significant decrease in the number of CD45/LCA + cells in grade III meningioma samples $(p=0.008$ comparing grade I with grade III) (Fig. 1B) with this decrease corresponding to a decrease in the number of CD3 + T lymphocytes $(\mathrm{p}=0.040)$ (Fig. 1C) - including a decrease in both CD4+ lymphocytes ( $p$ $=0.012)$ (Fig. 1D) and CD8+ lymphocytes $(\mathrm{p}=0.011)$ (Fig. 1E). To further characterize the lymphocyte infiltrate we stained the TMAs for the transcription factor FOXP3 that is a key transcription factor controlling $\mathrm{T}$ regulatory cell (Treg) development and function (Fig. 1F) as well as PD-1, an inhibitory cell surface receptor involved in the regulation of T-cell function (Fig. 1G). Interestingly, 
Table 1: Immune cell infiltrate in 291 cases of meningioma scored by Aperio digital algorithms (separated into low and high expressors - relative to the median score for each marker for the entire cohort; TMA $283 \& 285 \& 310)$

\begin{tabular}{|l|l|l|l|l|l|l|l|l|l|l|}
\hline \multirow{2}{*}{ Meningioma types } & \multirow{2}{*}{ \# of Cases } & \multicolumn{2}{l}{ LCA } & \multicolumn{3}{l|}{ CD3 } & \multicolumn{2}{l|}{ CD4 } \\
\cline { 3 - 12 } & & Low & High & Percent & Low & High & Percent & Low & High & Percent \\
\hline WHO grade I & 195 & 83 & 103 & $55.38 \%$ & 91 & 103 & $53.09 \%$ & 98 & 96 & $49.48 \%$ \\
\hline Meningothelial & 68 & 22 & 43 & & 31 & 37 & & 36 & 32 & \\
\hline Fibrous & 46 & 25 & 20 & & 22 & 24 & & 29 & 17 & \\
\hline Transitional & 52 & 20 & 31 & & 22 & 30 & & 23 & 29 & \\
\hline Angiomatous & 11 & 6 & 4 & & 6 & 5 & & 4 & 7 & \\
\hline Psammomatous & 9 & 3 & 3 & & 3 & 5 & & 2 & 6 & \\
\hline Secretory & 9 & 7 & 2 & & 7 & 2 & & 4 & 5 & \\
\hline & & & & & & & & & & \\
\hline WHO grade II & 73 & 41 & 31 & $43.06 \%$ & 39 & 34 & $46.58 \%$ & 35 & 37 & $51.39 \%$ \\
\hline Atypical & 68 & 36 & 31 & $\mathrm{p}=0.076$ & 38 & 30 & $\mathrm{p}=0.342$ & 32 & 35 & $\mathrm{p}=0.783$ \\
\hline Chordoid & 5 & 5 & 0 & & 1 & 4 & & 3 & 2 & \\
\hline & & & & & & & & & & \\
\hline WHO grade III & 23 & 17 & 6 & $26.09 \%$ & 16 & 7 & $30.43 \%$ & 18 & 5 & $21.74 \%$ \\
\hline Anaplastic & 23 & 17 & 6 & $\mathrm{p}=0.008$ & 16 & 7 & $\mathrm{p}=0.040$ & 18 & 5 & $\mathrm{p}=0.012$ \\
\hline
\end{tabular}

\begin{tabular}{|l|l|l|l|l|l|l|l|l|l|l|l|l|}
\hline CD8 & \multicolumn{3}{|c|}{ CD20 } & \multicolumn{3}{l|}{ FOXP3 } & \multicolumn{2}{l|}{ PD-1 } \\
\hline Low & High & Percent & Low & High & Percent & Low & High & Percent & Low & High & Percent \\
\hline 89 & 105 & $54.12 \%$ & 120 & 74 & $38.14 \%$ & 114 & 79 & $40.93 \%$ & 105 & 89 & $45.88 \%$ \\
\hline 29 & 39 & & 38 & 30 & & 42 & 26 & & 30 & 38 & \\
\hline 20 & 26 & & 35 & 11 & & 27 & 19 & & 25 & 21 & \\
\hline 21 & 31 & & 33 & 19 & & 26 & 25 & & 29 & 23 & \\
\hline 6 & 5 & & 5 & 6 & & 7 & 4 & & 8 & 3 & \\
\hline 5 & 3 & & 5 & 3 & & 5 & 3 & & 5 & 3 & \\
\hline 8 & 1 & & 4 & 5 & & 7 & 2 & & 8 & 1 & \\
\hline & & & & & & & & & & & \\
\hline 39 & 34 & $46.58 \%$ & 46 & 27 & $36.99 \%$ & 26 & 47 & $64.38 \%$ & 33 & 38 & $53.52 \%$ \\
\hline 37 & 31 & $\mathrm{p}=0.271$ & 43 & 25 & $\mathrm{p}=0.862$ & 24 & 44 & $\mathrm{p}=0.001$ & 29 & 37 & $\mathrm{p}=0.270$ \\
\hline 2 & 3 & & 3 & 2 & & 2 & 3 & & 4 & 1 & \\
\hline & & & & & & & & & & & \\
\hline 17 & 6 & $26.09 \%$ & 17 & 6 & $26.09 \%$ & 5 & 18 & $78.26 \%$ & 18 & 5 & $21.74 \%$ \\
\hline 17 & 6 & $\mathrm{p}=0.011$ & 17 & 6 & $\mathrm{p}=0.257$ & 5 & 18 & $\mathrm{p}=0.001$ & 18 & 5 & $\mathrm{p}=0.027$ \\
\hline
\end{tabular}

The numbers of LCA, CD3, CD4, CD8, CD20, and FOXP3 positive cells were counted using Aperio Imagescope software and the number of PD-1 positive cells was counted by visual microscopic review. The percentage of cases with low or high infiltration are presented for each marker $(<$ or $>$ the median score across all of the samples in the cohort for each marker, respectively).

there was an increase in Foxp3+ cells in WHO grade II atypical meningioma and an even greater increase in WHO grade III anaplastic meningioma (Fig. 1F; p = 0.001). We also noted a drop in the number of PD-1+ cells in anaplastic meningioma, a change that was restricted to the anaplastic cases (Fig. 1G; $p=0.027$ ). There was no significant difference in the number of $\mathrm{B}$ cells between the three grades of meningioma (Fig. 1H). There was a strong correlation between the number of CD45/LCA+ cells in each of the three cores from each case (Fig. S1A-C) suggesting limited heterogeneity in the samples and that the samples on the TMA are respresentative of the tumor sample. 


\section{PD-L1 is expressed in meningioma cells}

The decrease in infiltrating $\mathrm{T}$ lymphocytes in anaplastic meningioma suggested to us that there is an immunosuppressive tumor microenvironment in these high-grade tumors. Because PD-L1 expression by tumor cells can mediate the inhibition of local immune responses, we next assessed whether meningioma cells express PDL1 mRNA and protein. We detected the expression of PDL1 mRNA in meningioma tumors using RT-PCR on RNA extracted from 16 frozen meningioma samples of various grades (Table S5). In all samples, we detected a specific signal for PD-L1 (Fig. 2A). Because the pattern of signal detected by RT-PCR in tissues cannot exclude that PD-L1 was expressed in infiltrating immune cells, we performed both RT-PCR (Fig. 2B) and immunoblot analysis (Fig. $2 \mathrm{C}$ ) on four primary patient derived meningioma cell lines (MG2, 5, 6 and 8; Table S6) to determine if meningioma cells themselves express PD-L1. These cell lines had been cultured for 6 to 20 passages and therefore did not have residual lymphocytes. PD-L1 mRNA and protein was detected in each of these cell lines (Fig. 2B and 2C). The mRNA signal was substantially reduced following transfection of siRNA targeting PD-L1, supporting the specificity of the RT-PCR mRNA detection (Fig. S2A).

To confirm cell surface expression of PD-L1 on meningioma cells we next used FACS analysis. In two resections of atypical meningioma, we found that both CD45+CD33+ tumor infiltrating myeloid cells and the CD45- non-immune cells which include meningioma cells express PD-L1 (Fig. 2D and 2E). In addition, using two of the primary meningioma cell line cultures from the panel used for immunoblotting above (MG2 and MG8), we found that PD-L1 protein is expressed on the cell surface of these cells (Fig. 2F and G). A small subset (10\% in MG2 and 5\% in MG8) had detectably higher expression than the other cells in the population. Of note, PD-L1 mRNA was also detected in two additional primary meningioma cell lines (MG9 and MG10) and four established cell lines that are used in meningioma studies (Ben-Men-1, IOMM-Lee, F5 and CH157) (Fig. S2B).

To assess PD-L1 expression in tissue resection specimens and to correlate expression with meningioma grade in our TMA cohorts, we first developed and tested a custom-designed RNAscope probe (see Materials and Methods) for detecting human PD-L1 mRNA transcripts by in situ hybridization in FFPE tissues with 3,3'-Diaminobenzidine (DAB) used for signal visualization. A different fluorescence-labeled RNAscope probe for detecting PD-L1 was recently reported for the evaluation of breast cancer tissue [38]. With our probe we observed strong dot-like signals in cells from human tonsil and from Hodgkin lymphoma (HL) in which a subset of the cells are known to have high expression of PD-L1 (Fig. $2 \mathrm{H})$. In the same tissues we saw no signal with a negative control DapB probe (Fig. 2H). In addition, the PD-L1 RNAscope did not show a signal in a range of normal human tissues including human kidney and liver and lowlevel signal in infrequent cells in the leptomeninges (Fig. $\mathrm{S} 2 \mathrm{C})$.
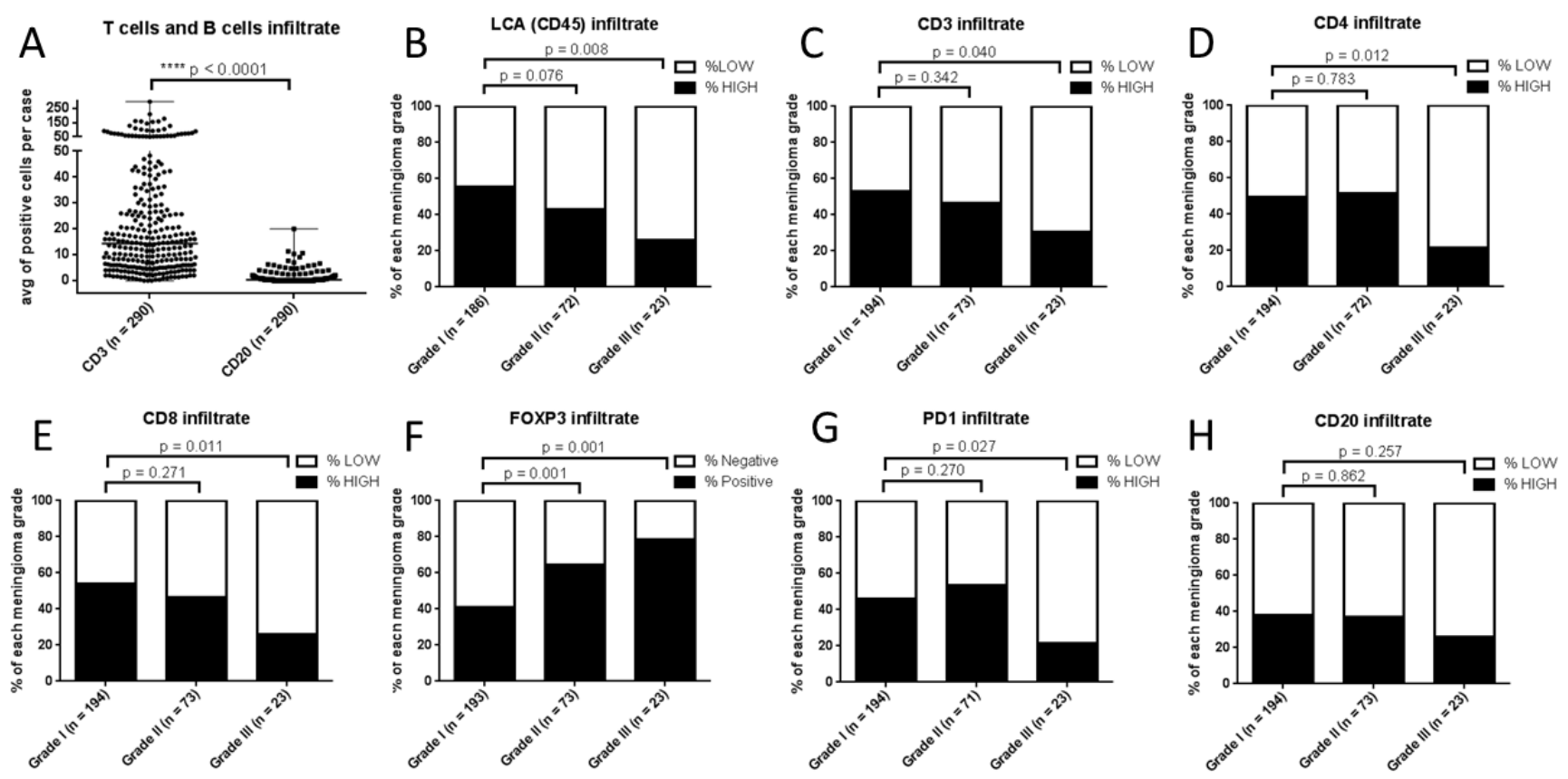

Figure 1: Characterization of immune cell infiltrate in meningioma. A, Scatter plot of CD3+ T cells and CD20+ B cells in 290 cases of meningioma from TMAs $283 \& 285$ and TMA310. The percentage of cases with low or high infiltration $(<$ or $>$ the median score across all of the samples in the cohort for each marker, respectively) for B, LCA/CD45; C, CD3; D, CD4; E, CD8; F, FOXP3; G, PD-1; and $\mathrm{H}, \mathrm{CD} 20$. 
To confirm expression of PD-L1 in meningioma, we stained our three meningioma TMAs with the PDL1 probe and detected signal in a portion of the samples - present at various levels in different cases (example images shown in Fig. 3A and S3). When expression was detected in a meningioma sample, signal was detected in most of the cells in the core, supporting that PD-L1 is expressed in meningioma cells (Fig. 3A and S3). Of note, 14 of the 16 frozen section samples examined by RT-PCR above had FFPE tissues in our TMAs and we observed a strong correlation $(r=0.5975 ; p=0.0240)$ between the PD-L1 signal detected by RT-PCR and the signal detected by RNAscope PD-L1 probe scored by an Aperio pixel counting Imagescope software (Table S5 and Fig S4A).

\section{PD-L1 expression is increased in WHO grade II and III anaplastic meningioma}

Having demonstrated that PD-L1 is expressed in meningioma tumors, we next proceeded to quantify the expression of PD-L1 mRNA (RNAscope) and protein (IHC) in our cohorts and investigated associations with WHO grade. We used the data from TMA283 \& 285 as our investigation cohort (Cohort one) (Fig. 3) and then confirmed the results using the cases from TMA310 as a validation cohort (Cohort two) (Fig. 4). To rigorously evaluate our findings, we quantified mRNA signal from the discovery cohort (TMA283 \& 285) using two digital analysis software - Aperio Imagescope which quantifies the number of positive pixels and intensity as

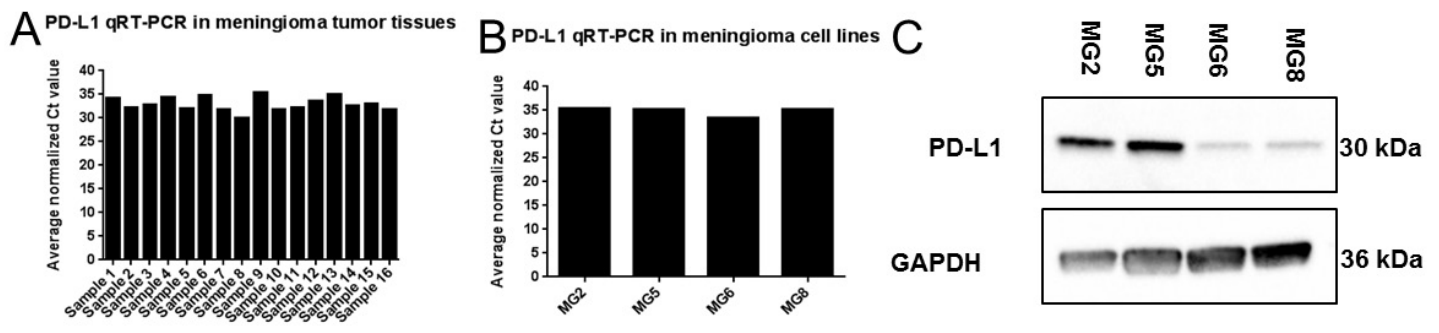

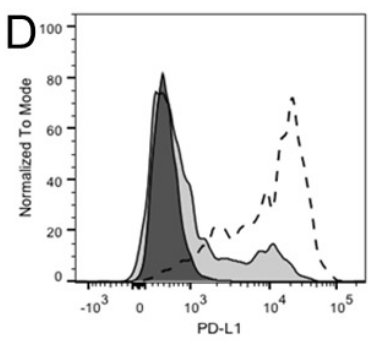

$\mathrm{H}$

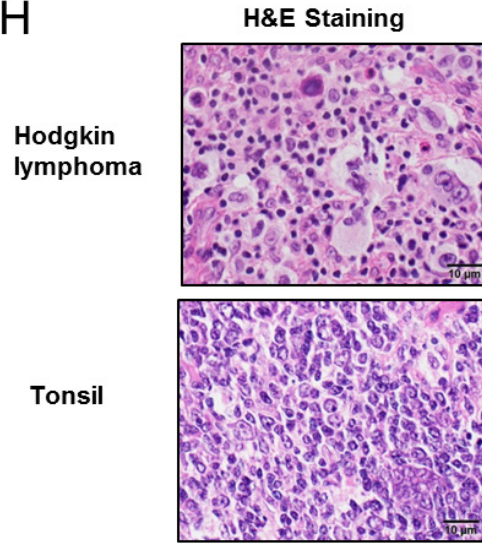

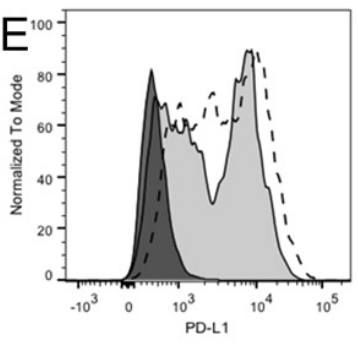

PD-L1 IHC 405.9A11)
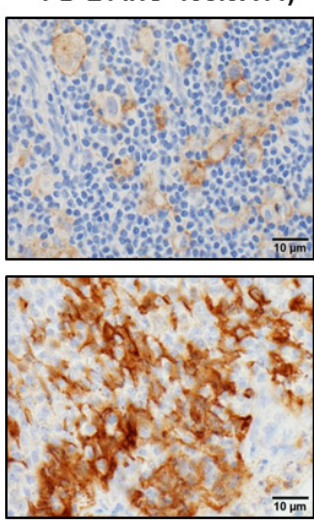

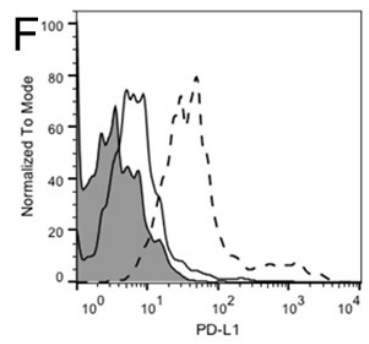

PD-L1 RNAscope

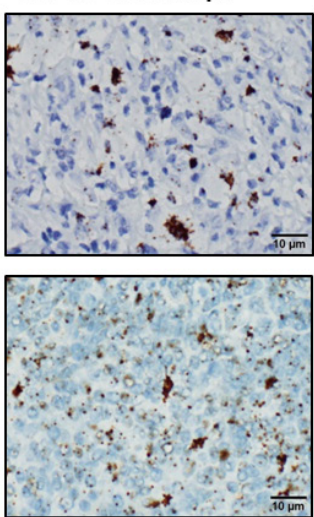

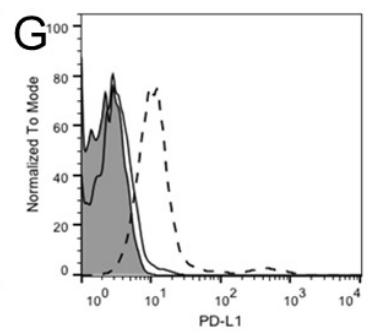

DapB RNAscope

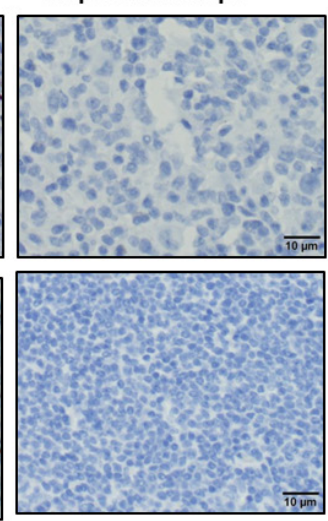

Figure 2: PD-L1 mRNA expression in meningioma tissues and cell lines. A, PD-L1 RT-PCR in 16 meningioma frozen tumor resection tissues; B, PD-L1 RT-PCR in 4 patient derived meningioma cell lines (MG2, MG5, MG6 and MG8); C, PD-L1 and GAPDH immunoblots of lysates from 4 primary meningioma cell lines (MG2, MG5, MG6 and MG8); D, PD-L1 FACS analysis of CD45+CD33+ tumor infiltrating myeloid cells in two meningioma tissue suspensions (Shaded histogram, tumor M17. Dotted histogram, tumor M20); E, PD-L1 FACS analysis of the CD45- cell population, which is the population which includes the tumor cells, in two meningioma tissue suspensions (Shaded histogram, tumor M17. Dotted histogram, tumor M20); PD-L1 FACS analysis in two primary meningioma cell lines MG2 (F) and MG8 (G) (Filled histogram, unlabeled. line histogram, isotype control. Dotted histogram, PD-L1).H, In situ hybridization of PD-L1 mRNA transcripts using a PD-L1-specific RNAscope probe and immunhistochemistry of PD-L1 using a PD-L1 specific antibody (405.9A11) in sections from FFPE tissues blocks of Hodgkin's lymphoma (HL) and human tonsil. 
well as the CellProfiler open source image quantification software [39] which permits cell by cell quantification of spot signals (dots per cell). To further validate our scoring algorithms we used Spotstudio ${ }^{\mathrm{TM}}$, a commercial software from the makers of RNAscope (Advanced Cell Diagnostics), to quantify the number of dots per cell in TMA310, in addition to Aperio Imagescope (pixel count) and CellProfiler (dot counting). Protein signal was quantified using a semi-quantitative assessment by visual review as well as using digital analysis software (Aperio Imagescope, positive pixel counting).

For RNAscope data analysis, in TMAs $283 \& 285$ there were a total of 173 meningioma cases (112 grade I, 43 grade II and 18 grade III). Fifteen cases had very low PPIB (which was used as a positive control) and were excluded from further analysis to avoid the possibility of false negative results. In total, 146 cases that were stained with our PD-L1 RNAscope probe could be evaluated by Aperio (Fig. 3C) and 119 cases by CellProfiler (Fig. 3D). By Aperio scoring (positive pixel counting), we found that compared to the expression in WHO grade I meningioma (median value $=102.5)$, there was significantly more PDL1 mRNA expression in both grade II (median value = $291.3 ; p=0.0012$ ) and grade III (median value $=326.0 ; p$ $=0.0229$ ) meningioma (Fig. S5A). Only $34.4 \%$ of WHO grade I meningiomas were PD-L1 mRNA high expressors
(Fig. 3C) defined as cases with scores above the median for the entire cohort, 179.9, Table S4) whereas $71.8 \%$ of WHO grade II meningiomas were high expressors $(\mathrm{p}<$ 0.001 ) and $82.4 \%$ of WHO grade III meningiomas were high expressors ( $p<0.001$ (Fig. 3C). Scoring the numbers of signal spots per cell in the same two TMAs (TMA283 \& 285) using the open source software CellProfiler analysis, we found similar results - that PD-L1 mRNA was highly expressed in $33.8 \%$ of WHO grade I, $70.3 \%$ WHO grade II, and $71.4 \%$ WHO grade III meningioma (Fig. 3D and S5B). The RNAscope Aperio (pixel counting) analysis and RNAscope CellProfiler (dot counting) analysis were highly correlated $(r=0.7652, p<0.0001)$ (Fig. S6A).

To determine if PD-L1 mRNA levels correlate with PD-L1 protein levels we performed immunohistochemistry on TMA283 and 285 using a Sinobiological antibody (see Materials and Methods). For IHC staining, we observed a similar pattern of PD-L1 expression when we quantified protein expression by Aperio positive pixel counting (Fig. S5C) or by visual light microscopic scoring (Fig. S5D). Compared to the expression in WHO grade I meningioma (median value $=51.8$ ), we noted significantly more PDL1 protein expression in both WHO grade II (median value $=96.7 ; \mathrm{p}<0.0001$ ) and WHO grade III (median value $=83.3 ; \mathrm{p}=0.0010)$ meningioma (Fig. S5C). Only $40.9 \%$ of WHO grade I meningioma were PD-L1 protein
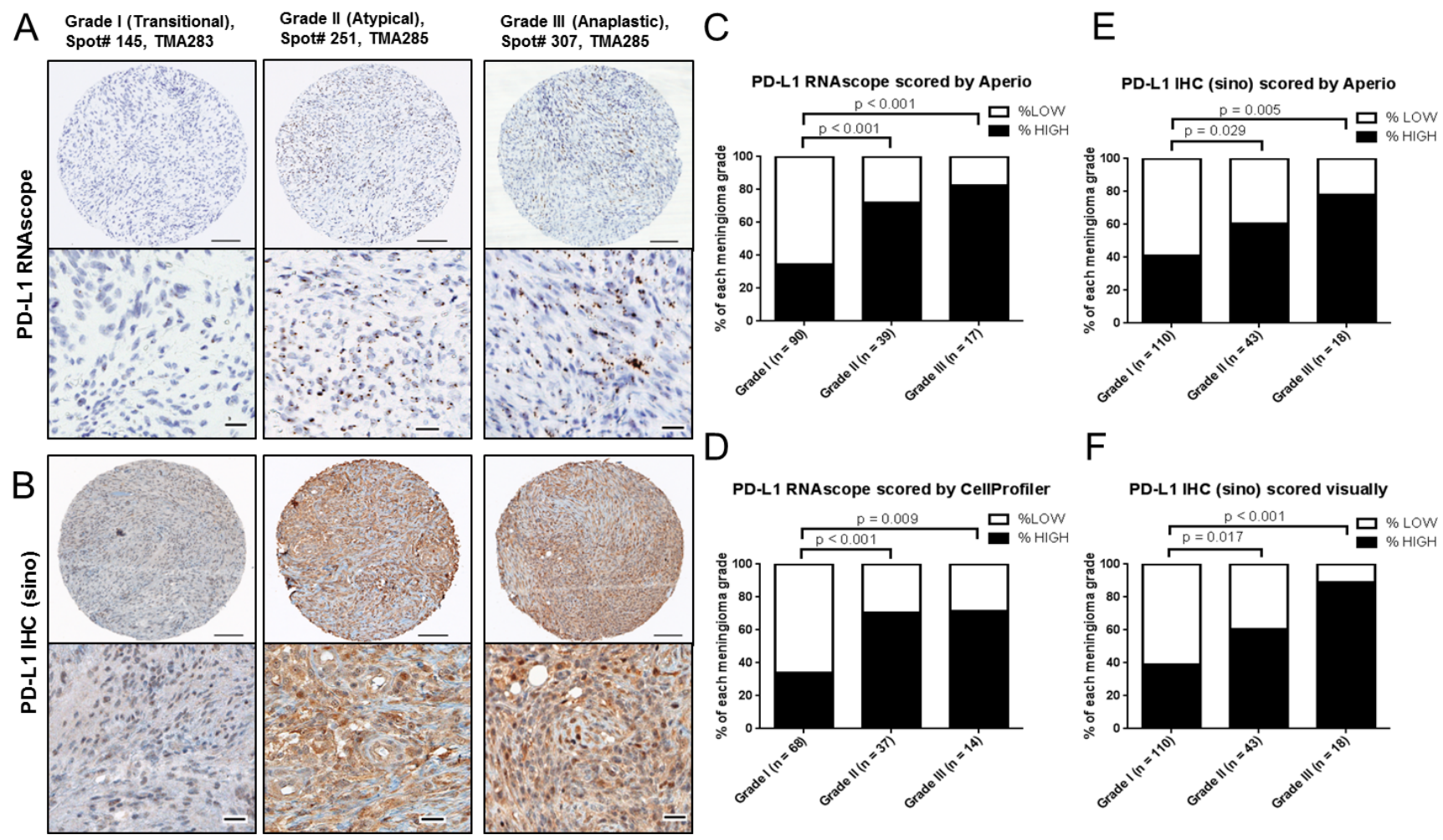

Figure 3: PD-L1 mRNA and protein expression in meningioma by grade. A, Representative images of PD-L1 RNAscope staining and B, PD-L1 IHC Sinobiological antibody staining from WHO grade I (transitional), WHO grade II (atypical) and WHO grade III (anaplastic) meningioma from TMAs 283 \& 285. Scale bar in low magnification, 100um; scale bar in high magnification, 20 um. PD-L1 RNAscope data was analyzed by C, Aperio and D, CellProfiler. PD-L1 IHC with a Sinobiological antibody was analyzed by E, Aperio and $\mathrm{F}$, visual light microscopy review. 
high expressors (defined as cases with scores above the median of 63.1 for the entire cohort; Table S4) whereas $60.5 \%$ of WHO grade II meningioma were high expressors ( $p=0.029)$ and $77.8 \%$ (14 of 18 cases) of WHO grade III meningiomas were high expressors $(p=0.005)$ (Fig. $3 \mathrm{E})$. Similar results were observed when the IHC stained slides were scored by visual review with high PD-L1 protein expression in $39.1 \% \mathrm{WHO}$ grade I, 60.5\% WHO grade II, and $88.9 \%$ WHO grade III meningioma (Fig. $3 \mathrm{~F})$. The IHC Aperio analysis and visual analysis were highly correlated $(r=0.8276, p<0.0001)$ (Fig. S6B). We noted a non-linear correlation between mRNA expression (RNAscope Aperio) and protein expression (by IHC (sino) Aperio or by IHC (sino) visual) in this TMA cohort ( $\mathrm{r}=$ 0.4890, $\mathrm{p}<0.0001 ; \mathrm{r}=0.3805, \mathrm{p}<0.0001$ ) (Fig. S6C and S6D).

To validate the strong association between WHO meningioma grade and both PD-L1 mRNA and protein expression we used a separate cohort (TMA310), and analyzed staining of the same RNAscope probe (Fig. 4A) and two different IHC antibodies - the same PD-L1 Sinobiological antibody used for IHC of TMAs $283 \& 285$ (Fig. 4B) and an additional PD-L1 antibody (405.9A11) (Fig. 4C). In TMA310 there were a total of 118 meningioma cases ( 83 grade I, 30 grade II and 5 grade III). Because this cohort is comprised of consecutive clinical cases over the last two years at Brigham and Women's Hospital and therefore reflects the frequency of cases in active clinical practice, only 5 of the 118 cases are WHO grade III anaplastic meningioma. So, for the analysis we grouped WHO grade II and III meningioma together as a "high-grade" group. Notably, the results in this cohort are very similar to those we observed with TMAs $283 \& 285$.

For RNAscope data analysis, 2 cases with very low PPIB were excluded from further analysis. For the Aperio analysis of PD-L1 RNAscope data 114 cases each having at least one informative core were available (Fig. S5E), for CellProfiler, 88 cases were available (Fig. S5F) and for Spotstudio ${ }^{\text {TM }} 90$ cases were available for analysis (Fig. S5G). The Aperio PD-L1 RNAscope (pixel counting) analysis revealed that compared to the expression in WHO grade I meningioma (median value $=142.0$ ), there was significantly more PD-L1 mRNA expressed in high-grade meningioma (median value = $355.0 ; p=0.0275$; (Fig. S5E). Only $43.2 \%$ of the WHO grade I meningioma were PD-L1 mRNA high expressors whereas $66.7 \%$ of the high-grade meningioma were high expressors ( $p=0.023$; Fig. 4D). The CellProfiler PD-L1 RNAscope (dot counting) analysis showed that PD-L1 mRNA was highly expressed in $41.0 \%$ of WHO grade I meningioma and in $70.4 \%$ of high-grade meningioma (Fig. 4E). Consistent with the results from both Aperio analysis and CellProfiler analysis (Figs. S5E and S5F), the analysis using Spotstudio ${ }^{\mathrm{TM}}$ (dot counting) software (Fig. S5G) showed that PD-L1 mRNA was highly expressed in $41.9 \%$ of WHO grade I meningioma and in $67.9 \%$ of
WHO grade II and III meningioma (Fig. 4F). There was very high correlation between the three methods (Aperio, CellProfiler and Spotstudio $\left.{ }^{\mathrm{TM}}\right)$ that we used to score PDL1 mRNA expression even though we measured staining intensity with pixel counts with Aperio Imagescope and individual dots per cell using CellProfiler and Spotstudio (Figs. S6E, S6F, S6G; r values between 0.7839 and $0.9105)$. Moreover, 12 of the cases that had frozen samples available and that we had previously analyzed with RTPCR also showed strong correlation between the RT-PCR values of PD-L1 and the scoring of PD-L1 RNAscope by Aperio $(r=0.5975)$, by CellProfiler $(r=0.7374)$ and by Spotstudio $^{\mathrm{TM}}(\mathrm{r}=0.7033)$ (Fig. S4 and Table S5). Similar to the limited heterogeneity seen for CD45/LCA+ cells, variability of PD-L1 mRNA expression between cores was low with high correlations between the three cores from each case (Fig. S1D-F).

Similar to the protein expression results observed in TMAs 283 \& 285, we noted a strong correlation between PD-L1 protein expression and WHO meningioma grade in the TMA310 validation cases. For this analysis we used two antibodies that recognize PD-L1 - PD-L1 (Sinobiological) (Fig. 4B) and PD-L1 (405.9A11) (Fig. 4C) antibodies and scored the staining using Aperio Imagescope software (Figs. S5H and S5I). For the PDL1 Sinobiological antibody, we observed that PD-L1 protein was highly expressed in $39.1 \%$ of WHO grade I meningioma but was highly expressed in $73.3 \%$ of higher grade meningioma (Fig. 4G and S5H). The PDL1 (405.9A11) antibody showed similar results, with PDL1 protein highly expressed in $40.7 \%$ of WHO grade I meningioma and in $72.7 \%$ of higher meningioma (Fig. $4 \mathrm{H}$ and S5I). The staining by these two antibodies was significantly correlated $(\mathrm{r}=0.6152, \mathrm{p}<0.0001)$ (Fig. $\mathrm{S} 6 \mathrm{H})$. We also noted a non-linear correlation between mRNA expression (RNAscope Aperio) and protein expression (by IHC with both PD-L1 (sino) and PD-L1 (405.9A11) antibodies) (Fig. S6I and S6J). Expression of PD-L1 mRNA and protein were not associated with a history of prior radiation therapy ( 237 patients with no history of radiation therapy and 40 with a history of prior radiation therapy).

\section{PD-L1 expression is not independently associated with outcome}

We performed cox proportional hazards modeling to investigate the impact of PD-L1 expression levels and grade on time to progression and all-cause mortality (in cohort one and two combined), and also assessed other clinical variable that have shown associations with grade such as age, performance status, extent of resection, and use of RT. On univariate analysis, grade III was highly correlated with both shorter time to progression $(\mathrm{HR}=$ $5.85, \mathrm{p}<0.0001)$ and all-cause mortality $(\mathrm{HR}=2.64$, 
$\mathrm{p}=0.02)$. Additional variables associated with time to progression were age at diagnosis $(\mathrm{HR}=1.03$ per year increase, $p=0.01)$, and gross total resection $(\mathrm{HR}=0.31$, $\mathrm{p}=0.0005)$. Additional variables associated with all-cause mortality included only gross total resection $(\mathrm{HR}=0.31$, $\mathrm{p}=0.0006$ ). After adjusting for grade, extent of surgical resection, and receipt of radiation therapy, neither Aperio pixel level or Cellprofiler score as associated with time to progression $(\mathrm{p}=\mathrm{NS})$. Similarly, after adjusting for grade, neither Aperio pixel level or CellProfiler score as associated with all-cause mortality $(\mathrm{p}=\mathrm{NS})$ suggesting that while PD-L1 may be a biomarker that may guide therapeutic intervention, that it is not, in this cohort, an independent prognostic indicator of poor outcome.

\section{DISCUSSION}

Our results demonstrate an alteration of the immune infiltrate of meningioma as these tumors become increasingly aggressive. In particular, we note a significant decrease in CD4+ and CD8+ T lymphocytes, a decrease in PD-1+ lymphocytes and an increase in FOXP3+ Treg lymphocytes in WHO grade III meningioma. Such a shift in the immune profile suggests that immune modulatory mechanisms may be important for the progression of meningioma and for the malignant behavior characteristic of WHO grade III meningioma. Notably, using several orthogonal analytical approaches we observe a significant increase in the expression of the mRNA and protein levels of the clinically relevant and actionable immune regulatory molecule PD-L1 in WHO grade III meningioma.

The pronounced clinical responses to immune blockade observed in other solid tumors such as advanced non-small-cell lung carcinoma, melanoma, and renal-cell carcinoma raises the possibility that testing of immune blockade strategies may be warranted in patients with aggressive high grade meningiomas that are associated with a poor clinical prognosis. It is reasonable to consider the work we present in this manuscript as hypothesis generating and that further validation in other cohorts will be optimal to determine that our analysis was not biased and that the results are generalizable. Prospective studies will be needed to validate the ability to assay PD-L1 as a biomarker in real time and to determine if there is an
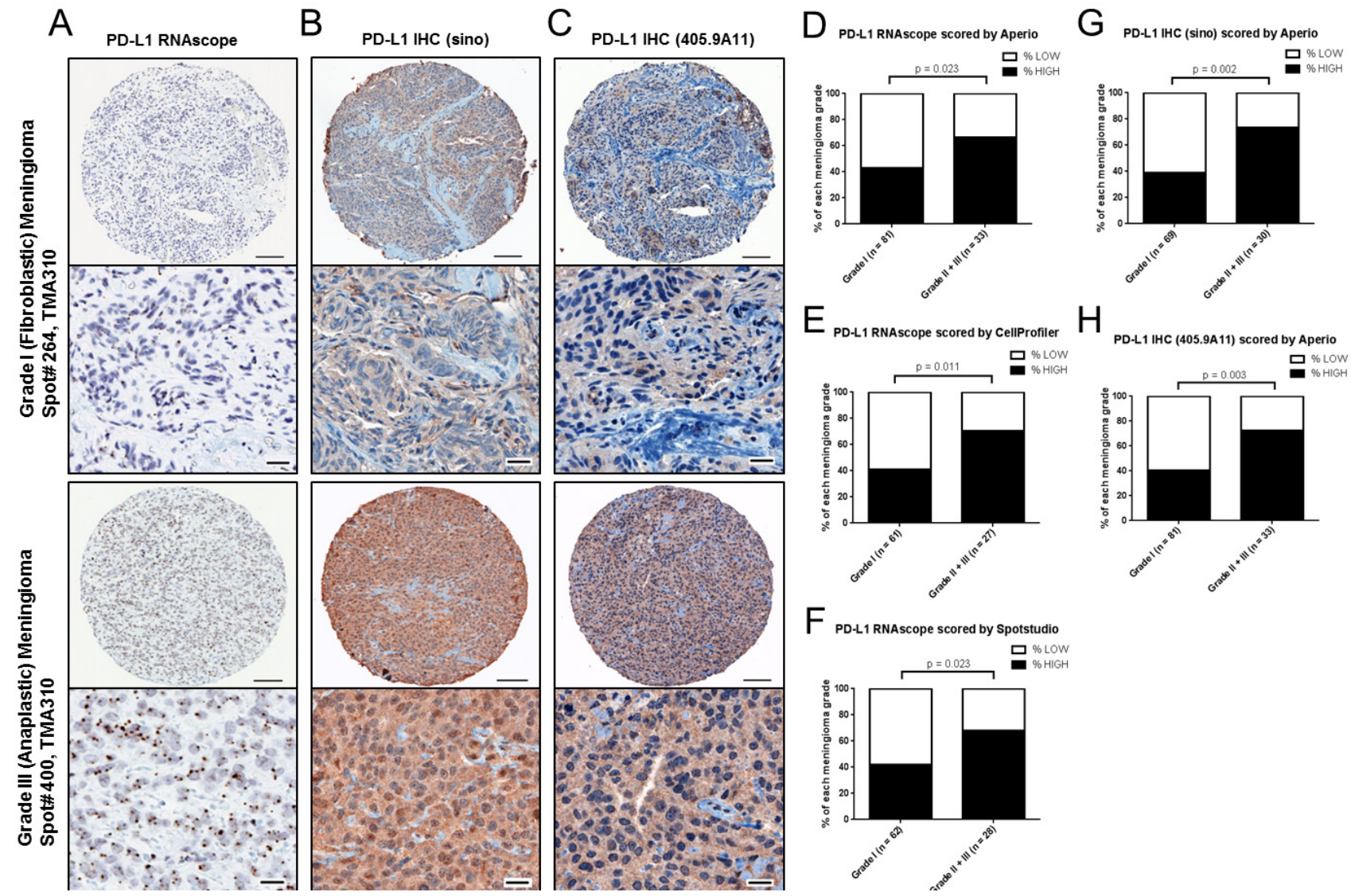

Figure 4: PD-L1 mRNA and protein expression in meningioma by grade in validation cohort. A, Representative images of PD-L1 RNAscope staining, B, PD-L1 IHC (Sinobiological) antibody staining and C, PD-L1 IHC (405.9A11) antibody staining from a WHO grade I (fibroblastic) meningioma and from a WHO grade III (anaplastic) meningioma from a validation TMA (TMA310). Scale bar in low magnification, 100um; scale bar in high magnification, $20 \mathrm{um}$. PD-L1 RNAscope data was analyzed by D, Aperio and E, CellProfiler and F, Spotstudio ${ }^{\mathrm{TM}}$. Aperio pixel scoring was used to score PD-L1 IHC with the G, (Sinobiological) antibody and with the H, (405.9A11) antibody. 
association between the level of PD-L1 expression and response to treatment. Our data suggests that while PDL1 expression is increased in higher grade meningioma, that it itself is not an independent prognostic indicator of outcome. Future studies with a larger number of samples and longer follow up will be needed to confirm this observation.

Prior studies of the immune infiltrate in meningiomas have focused predominantly on investigating the monocytic infiltrate of these tumors [40, 41] while others have characterized B and T lymphocyte infiltrates but on small sample sets $[42,43]$ (less than 30 samples). One study used flow cytometry analysis to characterize the lymphocyte population infiltrating 28 cases of meningioma [42] and demonstrated several important findings: that meningioma harbor both $\mathrm{T}$ and $\mathrm{B}$ lymphocytes that are antigen-experienced; that meningioma have CD4+ and CD8+ memory/effector $\mathrm{T}$ cells, regulatory $\mathrm{T}$ cells, and $\mathrm{T}$ cells expressing the immune checkpoint molecules PD-1; that these effector $\mathrm{T}$ cell populations are enriched relative to peripheral blood suggesting that certain $\mathrm{T}$ lymphocytes migrate to and reside in the tumors; and that the $\mathrm{T}$ lymphocytes infiltrating meningioma show phenotypes associated with 'exhaustion' suggesting that they may not be able to mount an effective immune response. Because this study used flow cytometry to characterize fresh meningioma tissues, the numbers of samples analyzed was relatively small (including only three WHO grade III meningiomas) compared to studies that use retrospective cohorts of FFPE fixed tissues. Hence, the authors could not assess if there are differences in the immune cell infiltrating populations of meningioma according to WHO grade and if there are significant changes in the expression of immune modulatory molecules in meningioma, particularly in the meningioma cells themselves. Our study, utilizing nearly 300 FFPE meningioma samples allowed us to address this important clinical issue by characterizing large numbers of samples and assessing the differences in the tumor infiltrating immune cells in high grade meningioma, the cases that are most likely to require treatment. A limitation of TMA based studies, on the other hand, is that the extracted TMA cores may miss potential heterogeneity that is present in the patient samples. Because we see high correlation between the scores of different cores for each patient samples, this suggests that the heterogeneity in meningioma may be less than that seen in other tumors, consistent with their relatively uniform morphological appearance seen by light microscopy.

Because antibodies for recognizing PD-L1 have some reported limitations [38, 44, 45], we developed a custom-designed RNAscope probe for detecting human PD-L1 mRNA transcripts. We thought that such a probe would have distinct advantages such as reduced background and increased specificity as well as readily quantifiable results. Indeed, using this probe we observed signal only in tissues known to harbor PD-L1 expressing cells like tonsil and Hodgkin's lymphoma and not in several other normal human tissues. Notably, even though we observed a significant correlation between PDL1 mRNA expression as detected by RNAscope in situ hybridization and PD-L1 protein expression as detected by IHC using two different anti-PD-L1 antibodies, the signal to noise ratio of the RNAscope probe was far better than that observed with IHC detection. This suggests that it may be important to test PD-L1 in situ hybridization probes alongside antibodies in clinical trial settings, especially in cohorts that are powered to capture correlations between clinical responses and PD-L1 expression levels. Such probes for immune regulatory molecules may become important biomarkers for predicting response to treatment.

Recent studies demonstrated the use of a different fluorescence-labeled RNAscope probe for detecting PDL1 in tumor tissues [38]. The DAB visualized probe that we used offers the advantage of readily correlating RNA expression with underlying histology using techniques and approaches that are customary to surgical pathologists. While proprietary algorithms have been streamlined to facilitate analysis of the RNAscope signals, we provide and validate an open-source CellProfiler image analysis software pipeline that can be readily optimized and implemented for quantifying RNAscope signals. This freely available software analysis pipeline could encourage the increased utilization of RNAscope in situ hybridization testing by research and clinical laboratories alike and could therefore facilitate the incorporation of RNAscope technologies into routine digital pathology workflows as well as into clinical trial correlative analyses.

\section{MATERIALS AND METHODS}

\section{Patient cohorts, clinical tissue samples, tissue microarrays and cell lines}

For this retrospective study, we used two sets of archival human meningioma formalin-fixed, paraffinembedded (FFPE) tissue specimens that we had collected from the Department of Pathology, Brigham and Women's Hospital, Harvard Medical School, Boston, Massachusetts. The study was approved by the Institutional Review Boards (IRBs) of Brigham and Women's Hospital and Dana Farber Cancer Institute, Harvard Medical School. Details are available in in the Supplemental Materials and Methods.

\section{RNAscope assay and evaluation}

In situ detection of PD-L1 transcripts in FFPE TMA samples was performed using the RNAscope ${ }^{\circledR}$ assay with Probe-Hs-PDL1-v2 (Cat\# 409671, Advanced 
Cell Diagnostics, USA) and RNAscope ${ }^{\circledR} 2.0 \mathrm{HD}$ Reagent Kit (BROWN) (Cat\# 310035, Advanced Cell Diagnostics, USA) following the protocols suggested by the manufacturer. See Supplemental Materials and Methods for details on staining and scoring with Aperio Imagescope, Spotstudio and Cell profiler.

\section{Immunohistochemistry and qRT-PCR assay}

Immunohistochemistry (IHC) was performed using a BOND III staining system (Leica Microsystems). The following primary antibodies were used in this study: LCA (1:600 dilution, Cat\# M0701; Dako, CA), CD3 (1:250 dilution, Cat\# A0452; Dako, CA), CD4 (1:80 dilution, Cat\# M7310; Dako, CA), CD8 (1:100 dilution, Cat\# M7103; Dako, CA), CD20 (Ready to Use, Cat\# N1502 RTU; Dako, CA), FOXP3 (1:50 dilution, Cat\# 320102; Biolegend, CA), PD-1 (1:300 dilution, Cat\# 315M-95; Cell Marque, CA), EMA (1:100 dilution, clone: E29, Cat\# M0613; DakoCytomation, CA), PD-L1 (1:36 dilution, Cat\# 10084-R015; Sinobiological, China), PD-L1 (1:125 dilution, 405.9A11; courtesy of Gordan Freeman lab DFCI)[46]. The staining protocols and scoring and details on the qRT-PCR are reported in the Supplemental Materials and Methods.

\section{Immunoblot}

Meningioma cell lines were harvested and lysed with RIPA buffer (Thermo Scientific, USA) and halt Protease Inhibitor Cocktail (Thermo Scientific, USA). Equal amounts of denatured protein sample were separated by SDS-PAGE and were then transferred to PVDF membranes using Trans-Blot ${ }^{\circledR}$ Turbo ${ }^{\mathrm{TM}}$ Midi PVDF Transfer Packs (Bio-Rad, USA) for immunoblot analysis. We used antibodies to recognize PD-L1 (1:200 dilution, Cat\# ab58810, Abcam, USA)[47, 48], GAPDH (1:500 dilution, Cat\# sc-32233, Santa Cruz, USA) as loading control. All protein bands were detected using Western Blotting Luminol Reagent (Cat\# sc-2048, Santa Cruz, USA).

\section{Fluorescence Activated Cell Sorting (FACS) Analysis}

Cryo-preserved meningioma single-cell suspensions were thawed at room temperature. The tumor suspensions were incubated on ice with anti-biotin microbeads (Miltenyi) and run through a magnetic column to remove excess debris from the cell slurry. The cells were split and stained with fluorochrome-labeled mAbs for CD33 (WM53, BD Biosciences), CD45 (HI30, BioLegend, PDL1 (29E.2A3, Biolegend) or an isotype control (mouse IgG2b, BioLegend) and fixed in 4\% paraformaldehyde.
Samples were run on either a FACSCalibur ${ }^{\mathrm{TM}}$ or FACSAria ${ }^{\mathrm{TM}}$ II (BD Biosciences) with CellQuest software and analyzed using FlowJo software (Tree Star).

\section{SiRNA transfection and Hematoxylin and Eosin (H\&E) staining}

Detailed information of siRNA transfection and (H\&E) staining are provided in the Supplemental Materials and Methods.

\section{Statistical analysis}

Details of analysis are provided in the Supplemental Materials and Methods.

\section{ACKNOWLEDGEMENTS}

The authors would like to thank members of the Santagata, Bradshaw and Agar labs for helpful discussions. The authors would like to thank Chungdak Namgyal for TMA construction and Terri Woo, Teri Bowman and the BWH/DFCI Specialized Histopathology Core for assistance with some of the immunohistochemistry staining. We thank Yuko G. Miki for guidance with cell culturing. We thank Dr. Mariano Viapiano and the Cooperative Human Tissue Network for tissue distributions for generating certain cell lines. We thank William G. Richards, Kristen K. Gill and the BWH tissue bank for access to frozen tissue samples as well as the BWH Division of Neuropathology under the leadership of Dr. Rebecca Folkerth for sample procurement. M.A.B. and A.E.C. were funded by National Institutes of Health RO1 GM089652 and H.H.S. and S.J.R. were supported by the Center for Immune Oncology at the Dana Farber Cancer Institute. This study was supported by Brain Science Foundation (S.S), the Jared Branfman Sunflowers for Life Fund for Pediatric Brain and Spinal Cancer Research (S.S), the V Foundation (S.S), King Abdulaziz City for Science and Technology (KACST), Saudi Arabia (M.A.) and National Institutes of Health RO1 GM089652 $(\mathrm{A}, \mathrm{E}, \mathrm{C})$.

\section{Conflict of interest}

S. Santagata and N. Agar are consultants and cofounders of Bayesian Diagnostics. There are no potential conflicts of interest disclosed by the other authors.

\section{EDITORIAL NOTE}

This paper has been accepted based in part on peerreview conducted by another journal and the authors' 
response and revisions as well as expedited peer-review in Oncotarget.

\section{REFERENCES}

1. Ostrom QT, Gittleman H, Farah P, Ondracek A, Chen Y, Wolinsky Y, Stroup NE, Kruchko C and Barnholtz-Sloan JS. CBTRUS statistical report: Primary brain and central nervous system tumors diagnosed in the United States in 2006-2010. Neuro Oncol. 2013; 15 Suppl 2:ii1-56.

2. Wiemels J, Wrensch M and Claus EB. Epidemiology and etiology of meningioma. J Neurooncol. 2010; 99(3):307314.

3. Pearson BE, Markert JM, Fisher WS, Guthrie BL, Fiveash JB, Palmer CA and Riley K. Hitting a moving target: evolution of a treatment paradigm for atypical meningiomas amid changing diagnostic criteria. Neurosurg Focus. 2008; 24(5):E3.

4. Claus EB, Bondy ML, Schildkraut JM, Wiemels JL, Wrensch M and Black PM. Epidemiology of intracranial meningioma. Neurosurgery. 2005; 57(6):1088-1095; discussion 1088-1095.

5. Kaur G, Sayegh ET, Larson A, Bloch O, Madden M, Sun MZ, Barani IJ, James CD and Parsa AT. Adjuvant radiotherapy for atypical and malignant meningiomas: a systematic review. Neuro Oncol. 2014; 16(5):628-636.

6. Perry A, Scheithauer BW, Stafford SL, Lohse CM and Wollan PC. "Malignancy" in meningiomas: a clinicopathologic study of 116 patients, with grading implications. Cancer. 1999; 85(9):2046-2056.

7. Yang SY, Park CK, Park SH, Kim DG, Chung YS and Jung HW. Atypical and anaplastic meningiomas: prognostic implications of clinicopathological features. J Neurol Neurosurg Psychiatry. 2008; 79(5):574-580.

8. Pasquier D, Bijmolt S, Veninga T, Rezvoy N, Villa S, Krengli M, Weber DC, Baumert BG, Canyilmaz E, Yalman D, Szutowicz E, Tzuk-Shina T, Mirimanoff RO and Rare Cancer N. Atypical and malignant meningioma: outcome and prognostic factors in 119 irradiated patients. A multicenter, retrospective study of the Rare Cancer Network. Int J Radiat Oncol Biol Phys. 2008; 71(5):13881393.

9. Palma L, Celli P, Franco C, Cervoni L and Cantore G. Longterm prognosis for atypical and malignant meningiomas: a study of 71 surgical cases. J Neurosurg. 1997; 86(5):793800 .

10. Clark VE, Erson-Omay EZ, Serin A, Yin J, Cotney J, Ozduman K, Avsar T, Li J, Murray PB, Henegariu O, Yilmaz S, Gunel JM, Carrion-Grant G, Yilmaz B, Grady C, Tanrikulu B, et al. Genomic analysis of non-NF2 meningiomas reveals mutations in TRAF7, KLF4, AKT1, and SMO. Science. 2013; 339(6123):1077-1080

11. Brastianos PK, Horowitz PM, Santagata S, Jones RT, McKenna A, Getz G, Ligon KL, Palescandolo E,
Van Hummelen P, Ducar MD, Raza A, Sunkavalli A, Macconaill LE, Stemmer-Rachamimov AO, Louis DN, Hahn WC, et al. Genomic sequencing of meningiomas identifies oncogenic SMO and AKT1 mutations. Nature genetics. 2013; 45(3):285-289.

12. Trofatter JA, MacCollin MM, Rutter JL, Murrell JR, Duyao MP, Parry DM, Eldridge R, Kley N, Menon AG, Pulaski $\mathrm{K}$ and et al. A novel moesin-, ezrin-, radixin-like gene is a candidate for the neurofibromatosis 2 tumor suppressor. Cell. 1993; 72(5):791-800.

13. Wellenreuther R, Kraus JA, Lenartz D, Menon AG, Schramm J, Louis DN, Ramesh V, Gusella JF, Wiestler OD and von Deimling A. Analysis of the neurofibromatosis 2 gene reveals molecular variants of meningioma. Am J Pathol. 1995; 146(4):827-832.

14. Ruttledge MH, Sarrazin J, Rangaratnam S, Phelan CM, Twist E, Merel P, Delattre O, Thomas G, Nordenskjold M, Collins VP and et al. Evidence for the complete inactivation of the NF2 gene in the majority of sporadic meningiomas. Nature genetics. 1994; 6(2):180-184.

15. Lekanne Deprez RH, Bianchi AB, Groen NA, Seizinger BR, Hagemeijer A, van Drunen E, Bootsma D, Koper JW, Avezaat CJ, Kley N and et al. Frequent NF2 gene transcript mutations in sporadic meningiomas and vestibular schwannomas. Am J Hum Genet. 1994; 54(6):1022-1029.

16. Schroeder RD, Angelo LS and Kurzrock R. NF2/merlin in hereditary neurofibromatosis 2 versus cancer: biologic mechanisms and clinical associations. Oncotarget. 2014; 5(1):67-77.

17. Reuss DE, Piro RM, Jones DT, Simon M, Ketter R, Kool M, Becker A, Sahm F, Pusch S, Meyer J, Hagenlocher C, Schweizer L, Capper D, Kickingereder P, Mucha J, Koelsche C, et al. Secretory meningiomas are defined by combined KLF4 K409Q and TRAF7 mutations. Acta Neuropathol. 2013; 125(3):351-358.

18. Abedalthagafi MS, Merrill PH, Bi WL, Jones RT, Listewnik ML, Ramkissoon SH, Thorner AR, Dunn IF, Beroukhim R, Alexander BM, Brastianos PK, Francis JM, Folkerth $\mathrm{RD}$, Ligon KL, Van Hummelen $\mathrm{P}$, Ligon $\mathrm{AH}$, et al. Angiomatous meningiomas have a distinct genetic profile with multiple chromosomal polysomies including polysomy of chromosome 5. Oncotarget. 2014; 5(21):10596-10606.

19. Smith MJ, O'Sullivan J, Bhaskar SS, Hadfield KD, Poke G, Caird J, Sharif S, Eccles D, Fitzpatrick D, Rawluk D, du Plessis D, Newman WG and Evans DG. Loss-of-function mutations in SMARCE1 cause an inherited disorder of multiple spinal meningiomas. Nature genetics. 2013; 45(3):295-298.

20. Lamszus K, Kluwe L, Matschke J, Meissner H, Laas R and Westphal M. Allelic losses at 1p, 9q, 10q, 14q, and $22 \mathrm{q}$ in the progression of aggressive meningiomas and undifferentiated meningeal sarcomas. Cancer Genet Cytogenet. 1999; 110(2):103-110.

21. Bhattacharjee MB, Armstrong DD, Vogel H and Cooley LD. Cytogenetic analysis of 120 primary pediatric brain 
tumors and literature review. Cancer Genet Cytogenet. 1997; 97(1):39-53.

22. Lee Y, Liu J, Patel S, Cloughesy T, Lai A, Farooqi H, Seligson D, Dong J, Liau L, Becker D, Mischel P, Shams S and Nelson S. Genomic landscape of meningiomas. Brain Pathol. 2010; 20(4):751-762.

23. Bigner SH, Mark J and Bigner DD. Cytogenetics of human brain tumors. Cancer Genet Cytogenet. 1990; 47(2):141154.

24. Domingues PH, Sousa P, Otero A, Goncalves JM, Ruiz L, de Oliveira C, Lopes MC, Orfao A and Tabernero MD. Proposal for a new risk stratification classification for meningioma based on patient age, WHO tumor grade, size, localization, and karyotype. Neuro Oncol. 2014; 16(5):735747.

25. Goutagny S, Nault JC, Mallet M, Henin D, Rossi JZ and Kalamarides M. High Incidence of Activating TERT Promoter Mutations in Meningiomas Undergoing Malignant Progression. Brain Pathol. 2014; 24(2):184-189.

26. Phan GQ, Yang JC, Sherry RM, Hwu P, Topalian SL, Schwartzentruber DJ, Restifo NP, Haworth LR, Seipp CA, Freezer LJ, Morton KE, Mavroukakis SA, Duray $\mathrm{PH}$, Steinberg SM, Allison JP, Davis TA, et al. Cancer regression and autoimmunity induced by cytotoxic $\mathrm{T}$ lymphocyte-associated antigen 4 blockade in patients with metastatic melanoma. Proc Natl Acad Sci U S A. 2003; 100(14):8372-8377.

27. Leach DR, Krummel MF and Allison JP. Enhancement of antitumor immunity by CTLA-4 blockade. Science. 1996; 271(5256):1734-1736.

28. Pardoll DM. The blockade of immune checkpoints in cancer immunotherapy. Nat Rev Cancer. 2012; 12(4):252-264.

29. Korman AJ, Peggs KS and Allison JP. Checkpoint blockade in cancer immunotherapy. Adv Immunol. 2006; 90:297339.

30. Reardon DA, Freeman G, Wu C, Chiocca EA, Wucherpfennig KW, Wen PY, Fritsch EF, Curry WT, Jr., Sampson JH and Dranoff G. Immunotherapy advances for glioblastoma. Neuro Oncol. 2014.

31. Topalian SL, Sznol M, McDermott DF, Kluger HM, Carvajal RD, Sharfman WH, Brahmer JR, Lawrence DP, Atkins MB, Powderly JD, Leming PD, Lipson EJ, Puzanov I, Smith DC, Taube JM, Wigginton JM, et al. Survival, durable tumor remission, and long-term safety in patients with advanced melanoma receiving nivolumab. J Clin Oncol. 2014; 32(10):1020-1030.

32. Topalian SL, Hodi FS, Brahmer JR, Gettinger SN, Smith DC, McDermott DF, Powderly JD, Carvajal RD, Sosman JA, Atkins MB, Leming PD, Spigel DR, Antonia SJ, Horn L, Drake CG, Pardoll DM, et al. Safety, activity, and immune correlates of anti-PD-1 antibody in cancer. The New England journal of medicine. 2012; 366(26):24432454

33. Brahmer JR, Tykodi SS, Chow LQ, Hwu WJ, Topalian SL,
Hwu P, Drake CG, Camacho LH, Kauh J, Odunsi K, Pitot HC, Hamid O, Bhatia S, Martins R, Eaton K, Chen S, et al. Safety and activity of anti-PD-L1 antibody in patients with advanced cancer. The New England journal of medicine. 2012; 366(26):2455-2465.

34. Ansell SM, Lesokhin AM, Borrello I, Halwani A, Scott EC, Gutierrez M, Schuster SJ, Millenson MM, Cattry D, Freeman GJ, Rodig SJ, Chapuy B, Ligon AH, Zhu L, Grosso JF, Kim SY, et al. PD-1 Blockade with Nivolumab in Relapsed or Refractory Hodgkin's Lymphoma. The New England journal of medicine. 2014.

35. Shtivelman E, Davies MQ, Hwu P, Yang J, Lotem M, Oren M, Flaherty KT and Fisher DE. Pathways and therapeutic targets in melanoma. Oncotarget. 2014; 5(7):1701-1752.

36. Taube JM, Klein AP, Brahmer JR, Xu H, Pan X, Kim JH, Chen L, Pardoll DM, Topalian SL and Anders RA. Association of PD-1, PD-1 ligands, and other features of the tumor immune microenvironment with response to antiPD-1 therapy. Clin Cancer Res. 2014.

37. Wang F, Flanagan J, Su N, Wang LC, Bui S, Nielson A, Wu X, Vo HT, Ma XJ and Luo Y. RNAscope: a novel in situ RNA analysis platform for formalin-fixed, paraffinembedded tissues. J Mol Diagn. 2012; 14(1):22-29.

38. Schalper KA, Velcheti V, Carvajal D, Wimberly H, Brown J, Pusztai L and Rimm DL. In situ tumor PD-L1 mRNA expression is associated with increased TILs and better outcome in breast carcinomas. Clin Cancer Res. 2014; 20(10):2773-2782.

39. Kamentsky L, Jones TR, Fraser A, Bray MA, Logan DJ, Madden KL, Ljosa V, Rueden C, Eliceiri KW and Carpenter AE. Improved structure, function and compatibility for CellProfiler: modular high-throughput image analysis software. Bioinformatics. 2011; 27(8):1179-1180.

40. Domingues PH, Teodosio C, Ortiz J, Sousa P, Otero A, Maillo A, Barcena P, Garcia-Macias MC, Lopes MC, de Oliveira C, Orfao A and Tabernero MD. Immunophenotypic identification and characterization of tumor cells and infiltrating cell populations in meningiomas. Am J Pathol. 2012; 181(5):1749-1761.

41. Domingues PH, Teodosio C, Otero A, Sousa P, Ortiz J, Macias Mdel C, Goncalves JM, Nieto AB, Lopes MC, de Oliveira C, Orfao A and Tabernero MD. Association between inflammatory infiltrates and isolated monosomy 22/ del(22q) in meningiomas. PLoS One. 2013; 8(10):e74798.

42. Fang L, Lowther DE, Meizlish ML, Anderson RC, Bruce JN, Devine L, Huttner AJ, Kleinstein SH, Lee JY, Stern JN, Yaari G, Lovato L, Cronk KM and O'Connor KC. The immune cell infiltrate populating meningiomas is composed of mature, antigen-experienced T and B cells. Neuro Oncol. 2013; 15(11):1479-1490.

43. Rossi ML, Cruz Sanchez F, Hughes JT, Esiri MM and Coakham HB. Immunocytochemical study of the cellular immune response in meningiomas. J Clin Pathol. 1988; 41(3):314-319. 
44. Velcheti V, Schalper KA, Carvajal DE, Anagnostou VK, Syrigos KN, Sznol M, Herbst RS, Gettinger SN, Chen L and Rimm DL. Programmed death ligand-1 expression in nonsmall cell lung cancer. Laboratory investigation; a journal of technical methods and pathology. 2014; 94(1):107-116.

45. Gadiot J, Hooijkaas AI, Kaiser AD, van Tinteren H, van Boven $\mathrm{H}$ and Blank $\mathrm{C}$. Overall survival and PD-L1 expression in metastasized malignant melanoma. Cancer. 2011; 117(10):2192-2201.

46. Choueiri TK, Fay AP, Gray KP, Callea M, Ho TH, Albiges L, Bellmunt J, Song J, Carvo I, Lampron M, Stanton ML, Hodi FS, McDermott DF, Atkins MB, Freeman GJ, Hirsch MS, et al. PD-L1 expression in nonclear-cell renal cell carcinoma. Ann Oncol. 2014; 25(11):2178-2184.

47. Rossille D, Gressier M, Damotte D, Maucort-Boulch D, Pangault C, Semana G, Le Gouill S, Haioun C, Tarte K, Lamy T, Milpied N, Fest T, for the Groupe Ouest-Est des Leucemies et Autres Maladies du S and for the Groupe Ouest-Est des Leucemies et Autres Maladies du S. High level of soluble programmed cell death ligand 1 in blood impacts overall survival in aggressive diffuse large B-Cell lymphoma: results from a French multicenter clinical trial. Leukemia. 2014.

48. Noman MZ, Desantis G, Janji B, Hasmim M, Karray S, Dessen P, Bronte V and Chouaib S. PD-L1 is a novel direct target of HIF-1alpha, and its blockade under hypoxia enhanced MDSC-mediated T cell activation. J Exp Med. 2014; 211(5):781-790. 